Pacific Journal of Mathematics

WHICH WEIGHTED SHIFTS ARE SUBNORMAL 


\section{WHICH WEIGHTED SHIFTS ARE SUBNORMAL}

\section{J. G. STAMPFLI}

Lei $H$ be a Hilbert space with orthonormal basis $\left\{f_{j}\right\}_{j=1}^{\infty}$. If the operator $T$ is defined on $H$ by $T f_{i}=a_{j} f_{i+1}$ for $i=$ $1,2, \cdots$, where $\left|a_{i}\right| \leqq\left|a_{i+1}\right| \leqq M$ for $i=1,2, \cdots$, then $T$ will be called a monotone shift. The first section of the paper examines some of the elementary properties of such operators.

Every monotone shift is hyponormal. The central portion of the paper aims at discovering which monotone shifts are subnormal. Necessary and sufficient conditions are given in terms of the $\left\{a_{i}\right\}$. These conditions make it easy to show that even the first four coefficients $\left(a_{1}<a_{2}<a_{3}<a_{4}\right)$ may "prevent" a shift from being subnormal. However, for any $a_{1}<a_{2}<a_{3}$ there does exist a monotone shift with these as its initial terms. In fact, the unique minimal one is constructed.

A complete description is given of subnormal monotone shifts for which $\left|a_{j_{0}}\right|=\left|a_{j_{0}+1}\right|$ for some $j_{0}$. The paper concludes with counter-examples constructed from the machinery developed.

We are tacitly assuming that $\lim _{j \rightarrow \infty}\left|a_{j}\right|$ exists, i.e., $T$ is a bounded operator. If $\left|a_{j}\right|=\left|a_{j+1}\right|$ for $j=1,2, \cdots$, then $T$ is (up to unitary equivalence) simply a multiple of the justly famous unilateral shift.

We recall that an operator $T$ on a Hilbert space $H$ is subnormal if it is the restriction of a normal operator to an invariant subspace. The terms "point", "continuous" and "residual spectrum" have their usual meaning and are designated by $\sigma_{P}(\cdot), \sigma_{\sigma}(\cdot)$ and $\sigma_{R}(\cdot)$ respectively.

THEOREM 1. Let $T$ be a monotone shift on $H$ where $A=$ $\lim _{j \rightarrow \infty}\left|a_{j}\right|$, then

(i) $\|T\|=A$

(ii) $\sigma_{R}(T)=\{z:|z|<A\}$

(iii) $\sigma_{P}\left(T^{*}\right)=\{z:|z|<A\}$

(iv) $\sigma_{o}(T)=\sigma_{o}\left(T^{*}\right)=\{z:|z|=A\}$.

Proof. Surely (i) is clear.

For $\left|z_{0}\right|<A$, consider the vector $g=\sum_{n=1}^{\infty} z_{0}^{n} b_{n} f_{n}$ where $b_{k}=1$ if $a_{k-1}=0, a_{k} \neq 0$ and $b_{n+1}=z_{0} b_{n} / \bar{a}_{n}$ for $n>k$. Since $\left|z_{0} / \bar{a}_{n}\right| \leqq r<1$ for $n$ sufficiently large, $g \in H$. But $\left(T^{*}-z I\right) g=0$ so $g$ is the desired eigenvector, proving (iii). The relation $b_{n+1}=z_{0} b_{n} / \bar{a}_{n}$ is necessary which implies the eigenvalue $z_{0}$ is of multiplicity one.

For any $z$, it is clear that $(T-z I) h \neq 0$ for $h \in H$, and $h \neq 0$.

Received January 26, 1965. Supported by the Office of Naval Research under contract Nonr (G)-00013-65. 
Now for an arbitrary operator, $z_{0} \in \sigma_{P}\left(T^{*}\right)$ implies $\bar{z}_{0} \in \sigma_{P}(T) \cup \sigma_{R}(T)$ and (ii) follows.

It is not hard to see that

$$
\sigma_{P}(T) \cap\{z:|z|=A\}=\phi=\sigma_{P}\left(T^{*}\right) \cap\{z:|z|=A\}
$$

and hence (iv) is proved.

REMARK. If one considered a two-sided monotone shift $T f_{j}=$ $a_{j} f_{j+1}$ for $j=0, \pm 1, \cdots$, then the above theorem is easily modified, the spectrum of $T$ now being the annulus $B \leqq|z| \leqq A$ where $A=$ $\lim _{j \rightarrow \infty}\left|\alpha_{j}\right|$ and $B=\lim _{j \rightarrow-\infty}\left|a_{j}\right|$.

THEOREM 2. If $T$ is a monotone shift then $T$ is hyponormal; that is $\|T g\| \geqq\left\|T^{*} g\right\|$ for $g \in H$.

Proof. Clear.

THEOREM 3. If $T$ is a monotone shift then $T$ is not a spectral operator, $($ for $T \neq 0)$.

Proof. Assume $T$ is a spectral operator; then $T^{*}$ is also a spectral operator which can be written as $S+N$ where $S$ is a sealar operator and $N$ a commuting quasi-nilpotent. By Theorem 1 we know that every point in the disc $|z|<\|T\|$ is an eigenvalue of $T^{*}$. Invoking Corollary 5 to Theorem 1 of [2] every point in the disc $|z|<\|T\|$ is an eigenvalue of $S$. But $S$ is similar to a normal operator which must have an uncountable number of distinct eigenvalues. Thus our original assumption was ill founded.

Next we would like explicitly exhibit a normal extension $B$ of $T$ assuming $T$ to be subnormal. The normal extension we construct will be minimal, (see [1], [3] for a discussion of subnormal operators). From now on we will assume $a_{i} \neq 0$ for $i=1,2, \cdots$. There is no real loss in generality if we do so. For if $a_{1}=a_{2}=\cdots=a_{n}=0$, then the subspace $M$ spanned by $\left\{\varphi_{1}, \cdots, \varphi_{n}\right\}$ is a reducing subspace of $T$ and moreover $T$ is normal on $M$. Hence we are really only concerned with what takes place in $M^{\perp}$. Let us designate the original basis for $H \equiv H^{(1)}$ by $\left\{\varphi_{j}^{(1)}\right\}_{j=1}^{\infty}$. We shall use the terms $a_{j}$ and $a_{j}^{(1)}$ interchangeably as a notational convenience. It will be to our advantage to assume that $\alpha_{j}^{(j)}>0$, for $j=1,2, \cdots$, and we may do this by simply rotating the $\varphi_{j}^{(1)}$ 's of the original basis by $e^{i \theta_{j}}$.

Now set $B^{*} \varphi_{j}^{(1)}=\alpha_{j-1}^{(1)} \varphi_{j-1}^{(1)}+b_{j}^{(2)} \varphi_{j}^{(2)}$ where $\left\|\varphi_{j}^{(2)}\right\|=1$ and $\varphi_{j}^{(2)}$ is orthogonal to $H^{(1)}$ for $j=1,2, \cdots$. Since $\left\|B^{*} \varphi_{j}^{(1)}\right\|^{2}=\left(a_{j-1}^{(1)}\right)^{2}+\left|b_{j}^{(2)}\right|^{2}=$ $\left\|B \varphi_{j}^{(1)}\right\|^{2}=\left(a_{j}^{(1)}\right)^{2}$ we may conclude that $\left|b_{j}^{(2)}\right|^{2}=\left(a_{j}^{(1)}\right)^{2}-\left(a_{j-1}^{(1)}\right)^{2},\left(a_{0}^{(1)}=\right.$ $0)$. We claim that the $\varphi_{j}^{(2)}$ 's are orthogonal, for

$$
b_{i}^{(2)} b_{j}^{(2)}\left(\varphi_{i}^{(2)}, \varphi_{j}^{(2)}\right)=\left(B^{*} \varphi_{i}^{(1)}, B^{*} \varphi_{j}^{(1)}\right)=\left(B \varphi_{i}^{(1)}, B \varphi_{j}^{(1)}\right)=0,
$$


when $i \neq j$. We are assuming for the moment that $b_{j}^{(2)} \neq 0$ for $j=1,2, \cdots$. Since the $\varphi_{j}^{(2)}$ 's are determined up to a scalar of modulus one we may take $b_{j}^{(2)}=\left[\left(a_{j}^{(1)}\right)^{2}-\left(a_{j-1}^{(1)}\right)^{2}\right]^{1 / 2}$. Now let us consider the possibility that $b_{j_{0}}^{(2)}=0$. This would mean $a_{j_{0}}^{(1)}=a_{j_{0}-1}^{(1)}$ or that $\left\|T^{*} \varphi_{j_{0}}^{(1)}\right\|=\left\|T \varphi_{j_{0}}^{(1)}\right\|$. But by Theorem 4 of [5] the set of vectors $\left\{f:\left\|T^{*} f\right\|=\|T f\|\right\}$ forms a closed invariant subspace of $T$. Thus $\left\|T \varphi_{k}^{(1)}\right\|=a_{k}^{(1)}=a_{j_{0}}^{(1)}$ for $k \geqq j_{0}$ and thus $b_{h}^{(2)}=0$ for $k \geqq j_{0}$.

We will designate by $H^{(2)}$ the space spanned by $\left\{\varphi_{j}^{(2)}\right)$ for $b_{j}^{(2)} \neq 0$. So far we know that $\left(B \varphi_{j}^{(2)}, \varphi_{j}^{(1)}\right)=b_{j}^{(2)}=\left[\left(a_{j}^{(1)}\right)^{2}-\left(a_{j-1}^{(1)}\right)^{2}\right]^{1 / 2}$. Now

$$
\begin{aligned}
\left(B \varphi_{j+1}^{(2)}, B \varphi_{j}^{(1)}\right) & =\left(B \varphi_{j+1}^{(2)}, T \varphi_{j}^{(1)}\right)=b_{j+1}^{(2)} \alpha_{j}^{(1)}=\left(B^{*} \varphi_{j+1}^{(2)}, B^{*} \varphi_{j}^{(1)}\right) \\
& =\left(B^{*} \varphi_{j+1}^{(2)}, \alpha_{j-1}^{(1)} \varphi_{j-1}^{(1)}+b_{j}^{(2)} \varphi_{j}^{(2)}\right) \\
& =\left(B^{*} \varphi_{j+1}^{(2)}, b_{j}^{(2)} \varphi_{j}^{(2)}\right)=b_{j}^{(2)}\left(\varphi_{j+1}^{(2)}, B \varphi_{j}^{(2)}\right)
\end{aligned}
$$

thus $\left(B \varphi_{j}^{(2)}, \varphi_{j+1}^{(2)}\right)=a_{j}^{(1)} b_{j+1}^{(2)} / b_{j}^{(2)}$. We set $a_{j}^{(2)} \equiv a_{j}^{(1)} b_{j+1}^{(2)} / b_{j}^{(2)}$. Since $\left(\varphi_{j}^{(2)}\right.$, $\left.B^{*} B \varphi_{k}^{(1)}\right)=0$ for $k \neq j-1$, it follows that

$$
\begin{aligned}
0 & =\left(B^{*} \varphi_{j}^{(2)}, B^{*} \varphi_{k}^{(1)}\right)=\left(B^{*} \varphi_{j}^{(2)}, a_{k-1}^{(1)} \varphi_{k-1}^{(1)}+b_{k}^{(2)} \varphi_{k}^{(2)}\right) \\
=\left(B^{*} \varphi_{j}^{(2)}, b_{k}^{(2)} \varphi_{k}^{(2)}\right) & =b_{k}^{(2)}\left(\varphi_{j}^{(2)}, B \varphi_{k}^{(2)}\right)
\end{aligned}
$$

or $\left(B \varphi_{k}^{(2)}, \varphi_{j}^{(2)}\right)=0$ for $k \neq j-1$. Thus $B \varphi_{j}^{(2)}=a_{j}^{(2)} \varphi_{j+1}^{(2)}+b_{j}^{(2)} \phi_{j}^{(1)}+f_{j}$ where $f_{j}$ is orthogonal to $H^{(1)} \oplus H^{(2)}$. We now wish to show that $f_{j}=0$ for $j=1,2, \cdots$. We observe that $\left(B f_{j}, B \varphi_{k}^{(1)}\right)=\alpha_{k}^{(1)}\left(f_{j}, B^{*} \phi_{k+1}^{(1)}\right)=0$ for all $j, k$ since $B^{*} \varphi_{k+1}^{(1)} \in H^{(1)} \oplus H^{(2)}$. But then

$$
\begin{aligned}
0 & =\left(B^{*} f_{j}, B^{*} \varphi_{j}^{(1)}\right)=\left(f_{j}, B\left(b_{j}^{(2)} \varphi_{j}^{(2)}+a_{j-1}^{(1)} \varphi_{j-1}^{(1)}\right)\right) \\
& =\left(f_{j}, B b_{j}^{(2)} \varphi_{j}^{(2)}\right)=b_{j}^{(2)}\left(f_{j}, a_{j}^{(2)} \varphi_{j+1}^{(2)}+b_{j}^{(2)} \varphi_{j}^{(1)}+f_{j}\right) \\
& =b_{j}^{(2)}\left\|f_{j}\right\|
\end{aligned}
$$

from which we may conclude $f_{j}=0$. Thus we have $B \varphi_{j}^{(2)}=a_{j}^{(2)} \varphi_{j+1}^{(2)}+b_{j}^{(2)} \varphi_{j}^{(1)}$ and since $\left\{\varphi_{j}^{(2)}\right\}$ is a basis for $H^{(2)}$ and $B$ is bounded by assumption, $B$ is now defined on $H^{(1)} \oplus H^{(2)}$.

It is perhaps worth while to write it down in matrix form on $H^{(1)} \oplus H^{(2)}$ :

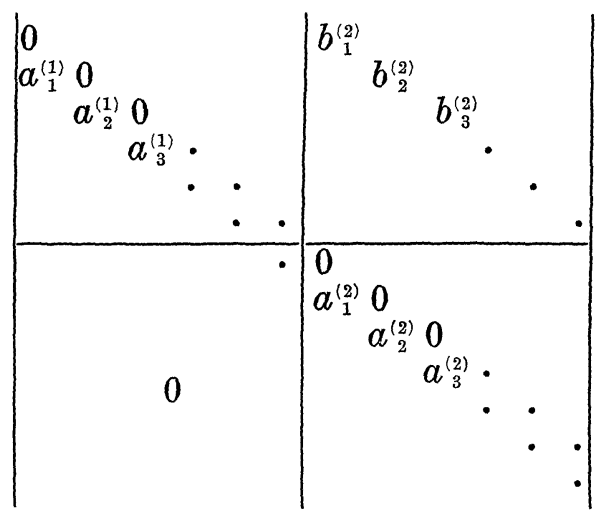


where $b_{j}^{(2)}=\left(\left(a_{j}^{(1)}\right)^{2}-\left(a_{j-1}^{(1)}\right)^{2}\right)^{1 / 2}$ and $b_{j}^{(2)} a_{j}^{(2)}=b_{j+1}^{(2)} a_{j}^{(1)}$. We have assumed that all the $a_{j}^{(1)} \mathrm{s} \neq 0$ and have shown that if $b_{j_{0}}^{(2)}=0$ then $b_{k}^{(2)}=0$ for $k \geqq j_{0}$. In this case, $H^{(2)}$ would be finite dimensional.

We will now assume $B$ has been defined on $H^{(1)}, \cdots, H^{(n)}$ where $\left\{\varphi_{j}^{(1)}\right\}, \cdots,\left\{\phi_{j}^{(n)}\right\}$ are the respective bases and $B \varphi_{j}^{(k)}=a_{j}^{(k)} \varphi_{j+1}^{(k)}+b_{j}^{(k)} \varphi_{j}^{(k-1)}$ for $k=2, \cdots, n$. We also note that all $b_{j}^{(k)} \neq 0$. That is, if $b_{1}^{(k)}, \cdots, b_{j_{k}}^{(k)}$ are nonzero and $b_{j_{k}+1}^{(k)}=0$ then $b_{j}^{(k)}=0$ for $j \geqq j_{k}+1$ and $H^{(k)}$ is spanned by $\left\{\varphi_{1}^{(k)}, \cdots, \varphi_{j_{k}}^{(k)}\right\}$. Further $a_{j}^{(k)} \neq 0$ for $j=1, \cdots, j_{k}-1$. The above can be considered to be the induction hypothesis.

We set $B^{*} \varphi_{j}^{(n)}=a_{j-1}^{(n)} \varphi_{j-1}^{(n)}+b_{j}^{(n+1)} \varphi_{j}^{(n+1)}$ for $j=1,2, \cdots$, where $\left\|\varphi_{j}^{(n+1)}\right\|=1$, and $\varphi_{j}^{(n+1)}$ is orthogonal to $H^{(n)} \oplus \cdots \oplus H^{(n)}$. Since $\left\|B^{*} \varphi_{j}^{(n)}\right\|=\left\|B \varphi_{j}^{(n)}\right\|$ we have $\left|b_{j}^{(n+1)}\right|^{2}=\left(a_{j}^{(n)}\right)^{2}+\left(b_{j}^{(n)}\right)^{2}-\left(a_{j-1}^{(n)}\right)^{2}\left(a_{0}^{(n)}=0\right.$ by definition). Now

$$
\begin{aligned}
\left(B^{*} \varphi_{i}^{(n)}, B^{*} \varphi_{j}^{(n)}\right) & =b_{i}^{(n+1)} b_{j}^{(n+1)}\left(\varphi_{i}^{(n+1)}, \varphi_{j}^{(n+1)}\right) \\
& =\left(B \varphi_{i}^{(n)}, B \varphi_{j}^{(n)}\right)=0 \text { for } i \neq j .
\end{aligned}
$$

Thus $\varphi_{i}^{(n+1)}$ and $\varphi_{j}^{(n+1)}$ are orthogonal for we may assume $b_{i}^{(n+1)}, b_{j}^{(n+1)}$ are nonzero else $\varphi_{i \text { or } j}^{(n+1)}$ would not be defined. Thus $b_{j}^{(n+1)}$ may be taken positive. Consider

$$
\begin{aligned}
\left(\varphi_{j+1}^{(n+1)}, B^{*} B \varphi_{j}^{(n)}\right) & =\left(\varphi_{j+1}^{(n+1)}, B^{*}\left(a_{j}^{(n)} \varphi_{j+1}^{(n)}+b_{j}^{(n)} \varphi_{j}^{(n-1)}\right)\right) \\
& =\left(\varphi_{j+1}^{(n+1)}, a_{j}^{(n)} b_{j+1}^{(n+1)} \varphi_{j+1}^{(n+1)}\right)=a_{j}^{(n)} b_{j+1}^{(n+1)} \\
& =\left(\varphi_{j+1}^{(n+1)}, B B^{*} \varphi_{j}^{(n)}\right)=\left(\varphi_{j+1}^{(n+1)}, B\left(a_{j-1}^{(n)} \varphi_{j-1}^{(n)}+b_{j}^{(n+1)} \varphi_{j}^{(n+1)}\right)\right) \\
& =\left(\varphi_{j+1}^{(n+1)}, b_{j}^{(n+1)} B \varphi_{j}^{(n+1)}\right) .
\end{aligned}
$$

This implies $B \varphi_{j}^{(n+1)}=b_{j}^{(n+1)} \varphi_{j}^{(n)}+a_{j}^{(n+1)} \varphi_{j+1}^{(n+1)}+f_{j}$ where $\left(f_{j}, \varphi_{j+1}^{(n+1)}\right)=0$ and $a_{j}^{(n+1)}$ satisfies the relation $a_{j}^{(n+1)} b_{j}^{(n+1)}=a_{j}^{(n)} b_{j+1}^{(n+1)}$, for $j=1,2, \cdots$.

Now let us clear up the zero, nonzero relation between the $a$ 's and the $b$ 's. By the induction hypothesis either all $a_{j}^{(n)}$ 's $\neq 0$ for $j=1,2, \cdots$, or there are a finite number and all but the last is nonzero. In either case assume $b_{k}^{(n+1)}=0$ and $b_{k+1}^{(n+1)} \neq 0$; then $a_{k}^{(n+1)} b_{k}^{(n+1)}=$ $0=a_{k}^{(n)} b_{k+1}^{(n+1)} \neq 0$ (for there are at least $k$ nonzero $a_{j}^{(n)}$ 's since the $\left.\operatorname{dim} H^{(n+1)} \leqq \operatorname{dim} H^{(n)}\right)$. This is impossible. Now assume $b_{j}^{(n+1)} \neq 0$ for $j=1,2, \cdots, k$, and $b_{j}^{(n+1)}=0$ for $j>k$, i.e., $H^{(n+1)}$ is $k$-dimensional. Then $a_{j}^{(n+1)}=a_{j}^{(n)} b_{j+1}^{(n+1)} / b_{j}^{(n+1)}$ is well defined for $j \leqq k$ and since $H^{(n)}$ was at least $k$-dimensional, $a_{j}^{(n)} \neq 0$ for $j=1, \cdots, k-1$; hence $a_{j}^{(n+1)} \neq 0$ for $j=1, \cdots, k-1$ as we wished to show.

Let us go back to the defining relation

$$
B \varphi_{j}^{(n+1)}=a_{j}^{(n+1)} \varphi_{j+1}^{(n+1)}+b_{j}^{(n+1)} \varphi_{j}^{(n)}+f_{j} .
$$

Since

$$
\left(\varphi_{i}^{(n+1)}, B^{*} B \varphi_{j}^{(n)}\right)=0 \quad \text { for } \quad i \neq j+1
$$

we have 


$$
\begin{aligned}
& \left(\varphi_{i}^{(n+1)}, B B^{*} \varphi_{j}^{(n)}\right)=\left(\varphi_{i}^{(n+1)}, b_{j}^{(n+1)}\left(a_{j}^{(n+1)} \varphi_{j+1}^{(n+1)}+b_{j}^{(n+1)} \varphi_{j}^{(n)}+f_{j}\right)\right) \\
+ & \left(\varphi_{i}^{(n+1)}, B a_{j-1}^{(n)} \varphi_{j-1}^{(n)}\right)=\left(\varphi_{i}^{(n+1)}, f_{j}\right)=0 \quad \text { for } i \neq j+1
\end{aligned}
$$

Now $\left(\varphi_{j+1}^{(n+1)}, f_{j}\right)=0$ by definition and hence $f_{j}$ is orthogonal to $H^{(1)} \oplus \cdots \oplus H^{(n+1)}$. But

$$
\begin{aligned}
\left(f_{j}, B^{*} B \varphi_{j}^{(n)}\right) & =\left(f_{j}, B^{*}\left(a_{j}^{(n)} \varphi_{j+1}^{(n)}+b_{j}^{(n)} \varphi_{j}^{(n-1)}\right)\right) \\
& =0=\left(f_{j}, B B^{*} \varphi_{j}^{(n)}\right) \\
& =\left(f_{j}, B\left(a_{j-1}^{(n)} \varphi_{j-1}^{(n)}+b_{j}^{(n+1)} \varphi_{j}^{(n+1)}\right)\right) \\
& =\left(f_{j}, b_{j}^{(n+1)} B \varphi_{j}^{(n+1)}\right) \\
& =\left(f_{j}, b_{j}^{(n+1)}\left(a_{j}^{(n+1)} \varphi_{j+1}^{(n+1)} b_{j}^{(n+1)} \varphi_{j}^{(h)}+f_{j}\right)\right) \\
& =b_{j}^{(n+1)}\left\|f_{j}\right\| \quad \text { and since } b_{j}^{(n+1)} \neq 0
\end{aligned}
$$

we conclude $f_{j}=0$. Thus $B \varphi_{j}^{(n+1)}=a_{j}^{(n+1)} \varphi_{j+1}^{(n+1)}+b_{j}^{(n+1)} \varphi_{j}^{(n)}$ where $b_{j}^{(n+1)} \neq 0$ for $j=1, \cdots,\left(\operatorname{dim} H^{(n+1)}\right.$ and $a_{j}^{(n+1)} \neq 0$ for $j=1, \cdots,\left(\operatorname{dim} H^{(n+1)}-1\right)$. It should help to present $B$ as an infinite matrix with respect to the basis $\left\{\varphi_{j}^{(n)}\right\}$ where $j=1,2, \cdots, \operatorname{dim} H^{(n)}$ and $n=1,2, \cdots$.

$$
B=\left|\begin{array}{llllll}
F^{(1)} & G^{(2)} & 0 & & \\
0 & F^{(2)} & G^{(3)} & & 0 & \\
0 & 0 & F^{(3)} & \cdot & & \\
& & & \cdot & \cdot & . \\
& 0 & & & & \cdot
\end{array}\right|
$$

where

$$
F^{(n)}=\left|\begin{array}{llllll}
0 & & & & & \\
a_{1}^{(n)} & 0 & & & & \\
& a_{2}^{(n)} & 0 & & & \\
& & a_{3}^{(n)} & \cdot & & \\
& & & \cdot & \cdot & \cdot \\
& & & & & \cdot
\end{array}\right|
$$

and

$$
G^{(n)}=\left|\begin{array}{llllll}
b_{1}^{(n)} & & & & & \\
& b_{2}^{(n)} & & & & \\
& & b_{3}^{(n)} & & & \\
& & & & \cdot & \\
& & & & & \cdot \mid
\end{array}\right|
$$

Now that we have a normal extension of $T$ in concrete form it is not difficult to read off some necessary conditions for the subnormality of $T$. In fact, it is clear that:

( I ) $\left(a_{j}^{(n)}\right)^{2}+\left(b_{j}^{(n)}\right)^{2} \geqq\left(a_{j-1}^{(n)}\right)^{2}$ for $n=2,3, \cdots$, and $j=1,2, \cdots$, (otherwise it would not be possible to define $b_{j}^{(n+1)}$ compatibly with the normality of $B$ ). 
(II) If $b_{j}^{(n)}=0$ then $b_{j+1}^{(n)}=0$ for $j=1,2, \cdots$, and $n=2,3, \cdots$, (else the equation $a_{j}^{(n)} b_{j}^{(n)}=a_{j}^{(n-1)} b_{j+1}^{(n)}$ could not be satisfied).

(III) There exists a constant $M$ such that $\left|\alpha_{j}^{(n)}\right| \leqq M$ and $\left|b_{j}^{(n)}\right| \leqq M$ for $n=2,3, \cdots$, and $j=1,2, \cdots$, (else the normal extension would not be bounded). These conditions are also sufficient. More precisely:

THEOREM 4. Let $\left\{a_{j}^{(1)}\right\}$ be a sequence such that $0<a_{1}^{(1)} \leqq a_{2}^{(1)} \leqq \cdots$ and define $T \phi_{j}^{(1)}=a_{j}^{(1)} \phi_{j+1}^{(1)}$ for $j=1,2, \cdots$, where $\left\{\phi_{j}^{(1)}\right\}_{j=1}^{\infty}$ is an orthonormal basis for $H^{(1)}$. The operator $T$ is subnormal if and only if

( I ) $\left(a_{j}^{(n)}\right)^{2}+\left(b_{j}^{(n)}\right)^{2}-\left(a_{j-1}^{(n)}\right)^{2} \geqq 0$

(II ) $b_{j}^{(n)}=0 \Rightarrow b_{j+1}^{(n)}=0$

(III) there exists a constant $M$ such that $\left|a_{j}^{(n)}\right| \leqq M$ and $\left|b_{j}^{(n)}\right| \leqq M$ for $n=2,3, \cdots$, and $j=1,2, \cdots$, where

$$
\left(b_{j}^{(n+1)}=+\left[\left(a_{j}^{(n)}\right)^{2}+\left(b_{j}^{(n)}\right)^{2}-\left(a_{j-1}^{(n)}\right)^{2}\right]^{1 / 2}\right.
$$

and $a_{j}^{(n+1)}=a_{j}^{(n)} b_{j+1}^{(n+1)} / b_{j}^{(n+1)}$ (if $b_{j_{0}}^{(n)}=0$ then $a_{j_{0}}^{(n)}$ is taken to be zero).

Proof. The necessity of (I), (II) and (III) has already been proved. In the other direction, since conditions (I) and (II) are satisfied the recursive definitions of the $\alpha_{j}^{(n+1)}$ and $b_{j}^{(n+1)}$ make sense. We now set $B \varphi_{j}^{(n)}=a_{j}^{(n)} \varphi_{j+1}^{(n)}+b_{j}^{(n)} \varphi_{j}^{(n-1)}$ where $\left\{\varphi_{j}^{(n)}\right\}$ is an orthonormal basis for $H^{(n)}$. If $b_{j_{0}}^{(n)}=0$ then $\varphi_{j_{0}}^{(n)}$ is omitted from the basis. With this definition it should be clear from the former construction that

$$
\left(B \varphi_{j}^{(n)}, B \varphi_{k}^{(m)}\right)=\left(B^{*} \varphi_{j}^{(n)}, B^{*} \varphi_{k}^{(m)}\right) \quad \text { for all } n, m, j, k \text {. }
$$

(Here as elsewhere all should be interpreted as vacuously covering the case when $\varphi_{j}^{(n)}$ has been omitted from the basis.) Thus $B$ is defined and satisfies $\|B x\|=\left\|B^{*} x\right\|$ on finite linear combinations of the basis elements. From (III) we may infer that $B$ is bounded: certainly $\|B\| \leqq 2 M$. Hence $B$ is defined and is normal on all of $\hat{H}=\sum \oplus H^{(n)}$.

We remark that the $a_{j}^{(1)}$ 's were taken to be real only as a notational convenience. To modify the theorem for an arbitrary monotone shift one need only add absolute value signs in the appropriate places.

Let $a_{1}, \cdots, a_{n}$ be given. If there exist elements $a_{n+1}, a_{n+2}, \cdots$, such that $T \varphi_{j}=a_{j} \varphi_{j+1}$ for $j=1,2, \cdots$, is a subnormal operator, we will call $T$ a subnormal completion of $a_{1}, \cdots, a_{n}$.

In Theorem 5 we will show that for any $a_{1}, a_{2}, a_{3}$ where $\left|a_{1}\right|<\left|a_{2}\right|<\left|a_{3}\right|$, there exists a subnormal completion. However, one can specify $a_{1}, a_{2}, a_{3}, a_{4}$, where $a_{1}<a_{2}<a_{3}<a_{4}$, in such a manner that they can not be completed to form a subnormal monotone shift. This can be easily done by making use of necessary condition (I). 
Consider $\left(a_{3}^{(2)}\right)^{2}+\left(b_{3}^{(2)}\right)^{2} \geqq\left(a_{2}^{(2)}\right)^{2}$ which is equivalent to:

$$
\left(a_{3}^{2}-a_{2}^{2}\right)+a_{3}^{2} \frac{a_{4}^{2}-a_{3}^{2}}{a_{3}^{2}-a_{2}^{2}} \geqq a_{2}^{2} \frac{a_{3}^{2}-a_{2}^{2}}{a_{2}^{2}-a_{1}^{2}}
$$

or

$$
a_{4}^{2} \geqq a_{3}^{2}+\frac{a_{1}^{2}}{a_{3}^{2}} \frac{\left(a_{3}^{2}-a_{2}^{2}\right)^{2}}{a_{2}^{2}-a_{1}^{2}}
$$

If $a_{4}$ is chosen so as not to satisfy (1) then clearly no choice of the remaining $a_{j}$ 's will make the resulting shift subnormal. For example, if $a_{1}=1, a_{2}=2^{1 / 2}, a_{3}=3^{1 / 2}$, then for $3^{1 / 2} \leqq a_{4}<(10 / 3)^{1 / 2}$ there exists no subnormal completion.

Theorem 5. Given $a_{1}, a_{2}, a_{3}$ where $0<\left|a_{1}\right|<\left|a_{2}\right|<\left|a_{3}\right|$ then there exists a subnormal completion of $a_{1}, a_{2}, a_{3}$. Moreover, if $S$ is any subnormal completion of $a_{1}, a_{2}, a_{3}$, then

$$
\begin{aligned}
\|S\|^{2} & \geqq 1 / 2\left[\left|a_{2}\right|^{2} \frac{\left|a_{3}\right|^{2}-\left|a_{1}\right|^{2}}{\left|a_{2}\right|^{2}-\left|a_{1}\right|^{2}}\right. \\
& \left.+\left(\left[\left|a_{2}\right|^{2} \frac{\left|a_{3}\right|^{2}-\left|a_{1}\right|^{2}}{\left|a_{2}\right|^{2}-\left|a_{1}\right|^{2}}\right]^{2}-4\left|a_{1}\right|^{2}\left|a_{2}\right|^{2} \frac{\left|a_{3}\right|^{2}-\left|a_{2}\right|^{2}}{\left|a_{2}\right|^{2}-\left|a_{1}\right|^{2}}\right)^{1 / 2}\right] .
\end{aligned}
$$

Further there is, up to unitary equivalence, exactly one subnormal completion for which equality holds.

Proof. We may take $a_{1}, a_{2}, a_{3}$ to be positive. The $a_{n}$ 's for the minimal completion are given by the following rule:

$$
a_{n}^{2} \frac{a_{n+1}^{2}-a_{n}^{2}}{a_{n}^{2}-a_{n-1}^{2}}=a_{n-1}^{2} \frac{a_{n}^{2}-a_{n-1}^{2}}{a_{n-1}^{2}-a_{n-1}^{2}}-\left(a_{n}^{2}-a_{n-1}^{2}\right) .
$$

Since $a_{n-1}^{2} /\left(a_{n-2}^{2}-a_{n-2}^{2}\right)>1$ the right side is strictly positive and $a_{n+1}>a_{n}$. The defining relation (2) is simply the necessary condition $\left(a_{j}^{(2)}\right)^{2}+\left(b_{j}^{(2)}\right)^{2}-\left(a_{j-1}^{(2)}\right)^{2} \geqq 0$ with equality holding.

Next we will find a more convenient expression for $a_{n}$. From (2) we have:

$$
\frac{a_{n+1}^{2}-a_{n}^{2}}{a_{n}^{2}-a_{n-1}^{2}}=\frac{a_{n-2}^{2}}{a_{n}^{2}} \cdot \frac{a_{n}^{2}-a_{n-1}^{2}}{a_{n-1}^{2}-a_{n-2}^{2}}
$$

and so by induction,

$$
\frac{a_{n+1}^{2}-a_{n}^{2}}{a_{n}^{2}-a_{n-1}^{2}}=\frac{a_{2}^{2} \cdot a_{1}^{2}}{a_{n}^{2} \cdot a_{n-1}^{2}} \cdot \frac{a_{3}^{2}-a_{2}^{2}}{a_{2}^{2}-a_{1}^{2}}=\frac{C}{a_{n}^{2} a_{n-1}^{2}}
$$

${ }^{1}$ Note that $a_{j}^{2}$ means $\left(a_{j}\right)^{2}$ not $a_{j}^{(2)}$ 
by definition of $C$. Thus

$$
a_{n+1}^{2}=a_{n}^{2}+C\left[\frac{1}{a_{n-1}^{2}}-\frac{1}{a_{n}^{2}}\right]
$$

and again by telescoping:

$$
a_{n+1}^{2}=a_{2}^{2}+C\left[\frac{1}{a_{1}^{2}}-\frac{1}{a_{n}^{2}}\right] .
$$

From (6) we can see that $\left\{a_{n}\right\}$ converges and we will shortly compute the limit.

Is the operator $T$ defined from the $a_{n}$ 's actually subnormal? Let us write down its normal extension.

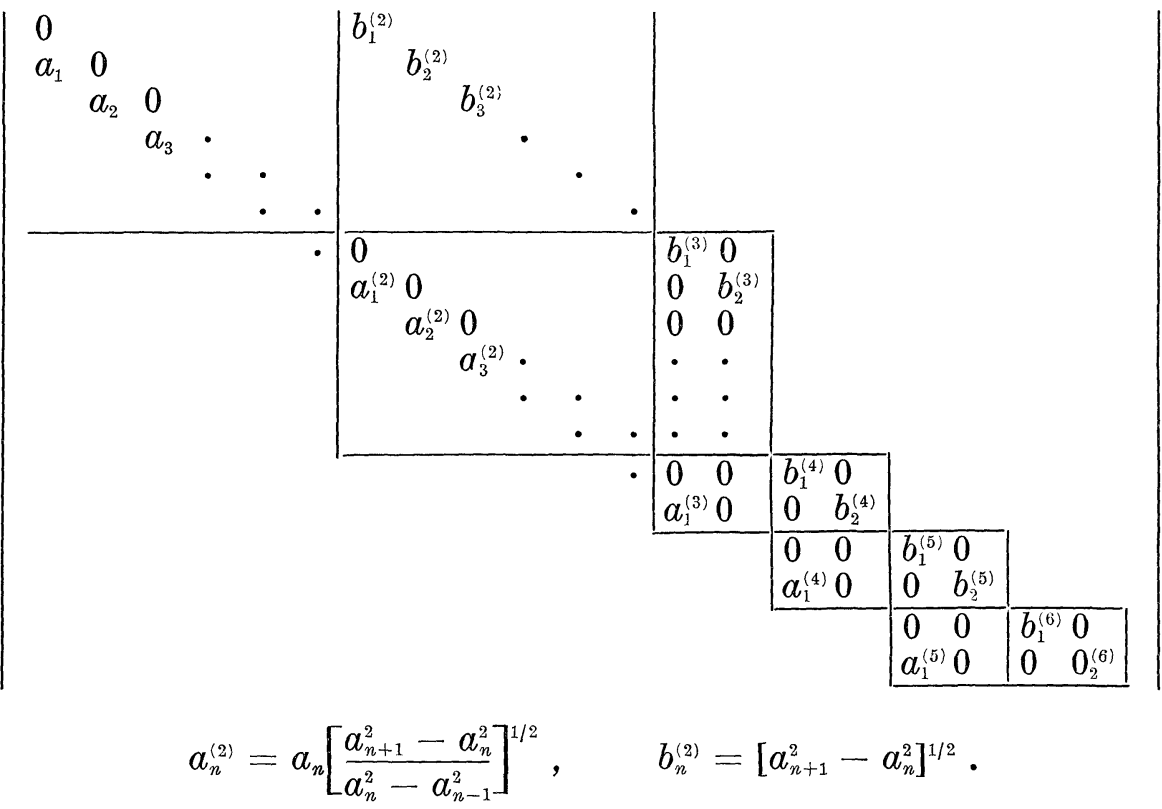

We see from (2) that

$$
a_{n}^{2} \frac{a_{n+1}^{2}-a_{n}^{2}}{a_{n}^{2}-a_{n-1}^{2}} \leqq a_{n-1}^{2} \frac{a_{n}^{2}-a_{n-1}^{2}}{a_{n-1}^{2}-a_{n-2}^{2}}
$$

and hence the $\alpha_{n}^{(2)}$ 's are bounded and obviously the $b_{n}^{(2)}$ 's are. Now if it can be shown that $a_{1}^{(k)}, b_{1}^{(k)}, b_{2}^{(k)}$, for $k=3,4, \cdots$, can be defined to satisfy the normality relations and moreover remain bounded then we are finished. The defining relations are:

and

(i ) $\quad a_{1}^{(n)} b_{1}^{(n)}=a_{1}^{(n-1)} b_{2}^{(n)}$

(ii) $\left(b_{1}^{(n+1)}\right)^{2}=\left(a_{1}^{(n)}\right)^{2}+\left(b_{1}^{(n)}\right)^{2}$

(iii) $\left(b_{2}^{(n+1)}\right)^{2}=\left(b_{2}^{(n)}\right)^{2}-\left(a_{1}^{(n)}\right)^{2}$. 
There is no difficulty in defining $a_{1}^{(n)}$ from (i) and $b_{1}^{(n+1)}$ from (ii). From (ii) we see that $b_{1}^{(n+1)}$ 's are increasing and $b_{1}^{(n+1)}>a_{1}^{(n)}$. Hence from (i) we have $1<b_{1}^{(n)} / a_{1}^{(n-1)}=b_{2}^{(n)} / a_{1}^{(n)}$ or $\left(b_{2}^{(n)}\right)^{2}-\left(a_{2}^{(n)}\right)^{2}-\left(a_{1}^{(n)}\right)^{2}>0$ so there is no obstacle to defining $b_{2}^{(n+1)}$ by (iii). Now to show boundedness. From (iii) we see $\left\{b_{2}^{(n)}\right\}$ is decreasing and thus converges which also implies that $\left\{a_{1}^{(n)}\right\}$ converges to zero. Combining (ii) and (iii) we have

$$
\left(b_{1}^{(n+1)}\right)^{2}-\left(b_{1}^{(n)}\right)^{2}=\left(b_{2}^{(n)}\right)^{2}-\left(b_{2}^{(n+1)}\right)^{2} .
$$

Thus

$$
\left(b_{1}^{(n+1)}\right)^{2}-\left(b_{2}^{(3)}\right)^{2}=\left(b_{2}^{(3)}\right)^{2}-\left(b_{2}^{(n+1)}\right)^{2}
$$

which implies $\left\{b_{1}^{(n)}\right\}$ converges since $\left\{b_{2}^{(n)}\right\}$ does. Now $B$ the normal extension of $T$ must be a bounded operator.

We will compute the norm of this completion from (6). Let $A=\lim _{j \rightarrow \infty}\left|a_{j}\right|^{2}=\|T\|^{2}$. Then (6) yields

$$
A=a_{2}^{2}+C\left[\frac{1}{a_{1}^{2}}-\frac{1}{A}\right]
$$

or

$$
A^{2}-\left(\alpha_{2}^{2}+C / \alpha_{1}^{2}\right) A-C=0 .
$$

Recalling that $C=a_{1}^{2} a_{2}^{2}\left[\left(a_{3}^{2}-a_{2}^{2}\right) /\left(a_{2}^{2}-a_{1}^{2}\right)\right]$ and selecting the proper root of the quadratic equation yields

$$
A=\frac{1}{2}\left[a_{2}^{2} \frac{a_{3}^{2}-a_{1}^{2}}{a_{2}^{2}-a_{1}^{2}}+\left\{\left[a_{2}^{2} \frac{a_{3}^{2}-a_{1}^{2}}{a_{2}^{2}-a_{1}^{2}}\right]^{2}-4 a_{1}^{2} a_{2}^{2} \frac{a_{3}^{2}-a_{2}^{2}}{a_{2}^{2}-a_{1}^{2}}\right\}^{1 / 2}\right]
$$

(the expression inside the radical is positive).

Before we show that the subnormal operator $T$ which we constructed to complete $a_{1}, a_{2}, a_{3}$ is minimal in norm, we will prove that it is an efficient subnormal completion in another sense. Let $S \varphi_{j}=c_{j} \varphi_{j+1}$ be any subnormal operator with $c_{i}=a_{i}$ for $i=1,2,3$. Rather than be inundated by absolute value signs we will assume that both the $a_{i}$ 's and the $c_{j}$ 's are positive.

Now the necessary condition (I)

$$
\left(\alpha_{j}^{(2)}\right)^{2}+\left(b_{j}^{(2)}\right)^{2}-\left(\alpha_{j-1}^{(2)}\right)^{2} \geqq 0
$$

applied to the operator $S$ yields the following analogue to (2):

$$
c_{n}^{2} \frac{c_{n+1}^{2}-c_{n}^{2}}{c_{n}^{2}-c_{n-1}^{2}} \geqq c_{n-1}^{2} \frac{c_{n}^{2}-c_{n-1}^{2}}{c_{n-1}^{2}-c_{n-2}^{2}}-\left(c_{n}^{2}-c_{n-1}^{2}\right)
$$

and if we argue as before we can reduce this to 


$$
c_{n+1}^{2} \geqq a_{2}^{2}+C\left[\frac{1}{a_{1}^{2}}-\frac{1}{c_{n}^{2}}\right] .
$$

If the completion $c_{n}$ is different from the one given above then for some first $k$ we have $c_{k}>a_{k}$ (it can not be less by the necessary condition (I)). But then we have

$$
c_{k+1}^{2} \geqq a_{2}^{2}+C\left[\frac{1}{a_{1}^{2}}-\frac{1}{c_{k}^{2}}\right]>a_{2}^{2}+C\left[\frac{1}{a_{1}^{2}}-\frac{1}{a_{k}^{2}}\right]=a_{k+1}^{2} .
$$

Hence $c_{j}>a_{j}$ for $j \geqq k$.

Now we will prove the last statement of the theorem. Let $S$ and $c_{j}$ be as above. Let $k$ be the smallest integer such that $a_{k+2}=c_{k+2}$ but $a_{k+3}<c_{k+3}$. Using the necessary condition (I) we arrive at

$$
a_{n+1}^{2}=a_{n}^{2}+D\left[\frac{1}{a_{n-1}^{2}}-\frac{1}{\alpha_{n}^{2}}\right]
$$

and

$$
a_{n+1}^{2} \geqq c_{n}^{2}+\hat{D}\left[\frac{1}{c_{n-1}^{2}}-\frac{1}{c_{n}^{2}}\right]
$$

for $n \geqq k+3$ where

$$
D=a_{k+2}^{2} a_{k+1}^{2} \frac{a_{k+3}^{2}-a_{k+2}^{2}}{a_{k+2}^{2}-a_{k+1}^{2}} \quad \text { and } \quad \hat{D}=c_{k+2}^{2} c_{k+1}^{2} \frac{c_{k+3}^{2}-c_{k+2}^{2}}{c_{k+2}^{2}-c_{k+1}^{2}} .
$$

These are obtained by a restricted telescoping of (3) and (9). Since $c_{k+1}=a_{k+1}, c_{k+2}=a_{k+2}$ and $c_{k+3}>a_{k+3}$, certainly $\hat{D}>D$. Now from (11a) and (11b) we obtain

$$
\alpha_{n+1}^{2}=\alpha_{k+2}^{2}+D\left[\frac{1}{\alpha_{k+1}^{2}}-\frac{1}{\alpha_{n}^{2}}\right]
$$

and

$$
c_{n+1}^{2} \geqq c_{n}^{2}+\hat{D}\left[\frac{1}{c_{k+1}^{2}}-\frac{1}{c_{n}^{2}}\right] \quad \text { for } \quad n \geqq k+3 \text {. }
$$

If we let $n \rightarrow \infty$ in (12a) and (12b) we obtain

$$
A=a_{k+2}^{2}+D\left[\frac{1}{a_{k+1}^{2}}-\frac{1}{A}\right]
$$

and

$$
\hat{A} \geqq c_{k+2}^{2}+\hat{D}\left[\frac{1}{a_{k+1}^{2}}-\frac{1}{\hat{A}}\right]
$$


or

$$
\hat{A} \geqq a_{k+2}^{2}+\hat{D}\left[\frac{1}{a_{k+1}^{2}}-\frac{1}{\hat{A}}\right]
$$

Now in (13a) the solution $A$ is a strictly increasing function of $D$ and since $\hat{D}>D$ we must have $\hat{A}>A$. But $\|T\|^{2}=A<\hat{A}=\|S\|^{2}$ and thus we have shown there is exactly one subnormal completion of $a_{1}, a_{2}, a_{3}$ with the norm given by (8) (again we note that operators are specified up to unitary equivalence only).

REMARK. The above construction of a subnormal completion of $a_{1}, a_{2}, a_{3}$ can be used in slightly more general circumstances. It has been pointed out that $a_{1}, a_{2}, a_{3}, a_{4}$ may not have a subnormal completion. If however $a_{4}$ satisfies the necessary condition

$$
a_{4}^{2} \geqq a_{3}^{2}+a_{1}^{2} \frac{\left(a_{3}^{2}-a_{2}^{2}\right)^{2}}{a_{2}^{2}-a_{1}^{2}}
$$

then one can obtain a subnormal completion by defining $a_{n}$ for $n \geqq 5$ as in (2). When one has $a_{1}, \cdots, a_{5}$ this definition does not work for reasons sufficiently complicated to remain unmentioned here.

Theorem 6. Let $T$ be a monotone shift, $T \varphi_{j}=a_{j} \varphi_{j+1}$. Assume further that

(i) $a_{j} \neq 0$ for $j=1,2, \cdots$

(ii) $T$ is subnormal

(iii) $\left|a_{k}\right|=\left|a_{k+1}\right|$ for some $k$ then $\left|a_{j}\right|=\left|a_{j+1}\right|$ for $j=2,3, \cdots$, and $a_{1}$ is arbitrary.

Proof. We will assume that the $a_{j}$ 's are positive. Let $k$ be the smallest integer such that $a_{k}=a_{k+1}$. If $k \leqq 2$ then by Theorem 4 of [5] we are through. For the case $k \geqq 3$ we now make use of Theorem 4. We observe that

$$
a_{k}^{(2)}=a_{k}\left[\frac{a_{k+1}^{2}-a_{k}^{2}}{a_{k}^{2}-a_{k-1}^{2}}\right]^{1 / 2}=0
$$

and

$$
\boldsymbol{a}_{k-1}^{(2)}=\boldsymbol{a}_{k-1}^{2}\left[\frac{\boldsymbol{a}_{k}^{2}-\boldsymbol{a}_{k-1}^{2}}{a_{k-1}^{2}-a_{k-1}^{2}}\right]^{1 / 2} \neq 0
$$

and

$$
b_{k}^{(2)}=\left[a_{k}^{2}-a_{k-1}^{2}\right]^{1 / 2} .
$$

Now a necessary condition for the subnormality of $T$ is just that 


$$
\begin{aligned}
0 & \leqq\left(a_{k}^{(2)}\right)^{2}+\left(b_{k}^{(2)}\right)^{2}-\left(a_{k-1}^{(2)}\right)^{2} \\
& =\left(a_{k}^{2}-a_{k-1}^{2}\right)-\frac{a_{k-1}^{2}}{a_{k-1}^{2}-a_{k-2}^{2}}\left(a_{k}^{2}-a_{k-1}^{2}\right) .
\end{aligned}
$$

But that implies $\left(a_{k-1}^{2}\right) / a_{k-1}^{2}\left(a_{k-2}^{2}\right) \leqq 1$ or $a_{k-2}=0$ contrary to hypothesis. This still leaves the existence of monotone shifts unsettled for $a_{1}<a_{2}=a_{j}$ for $j=2,3, \cdots$. We will show that these are all subnormal by writing down the subnormal extension. (The reader may do this by simply following the "directions" in Theorem 4.)

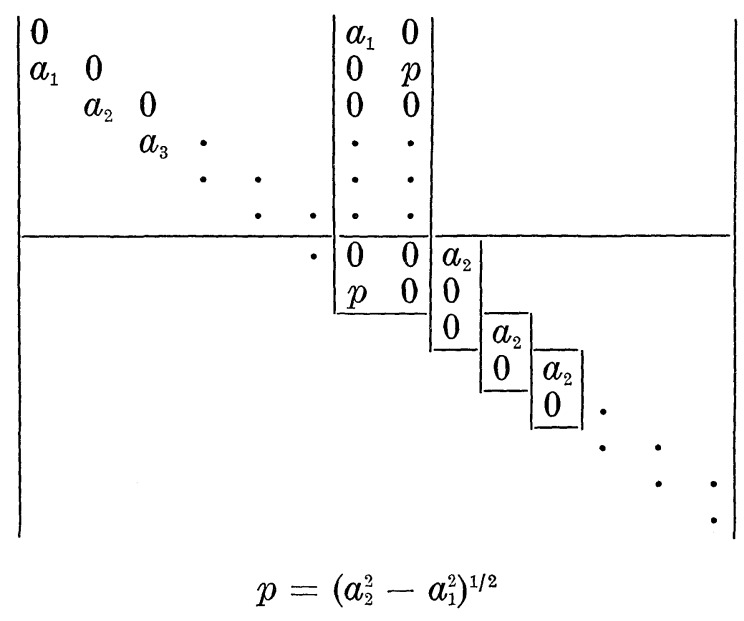

CoROLlaRy. The space $H^{(2)}$ is either infinite dimensional or $\operatorname{dim} H^{(2)} \leqq 2$. This is just another way of stating Theorem (6).

REMARK. This corollary has an analogue for the spaces $H^{(n)}$. We state without proof that either $H^{(n)}$ is infinite dimensional or $\operatorname{dim} H^{(n)} \leqq n$. In the proof of Theorem 5 we constructed a subnormal operator where $\operatorname{dim} H^{(3)}=2$. Given $a_{1}, a_{2}, a_{3}, a_{4}$, where $a_{4}$ satisfies the necessary condition (I), the construction yields an operator where $\operatorname{dim} H^{(3)}=3$.

Using the last theorem we can construct several counter-examples. In [4] we encounter the following:

THEOREM. If $T$ is hyponormal and $T^{n}$ is normal for some integer $n$ then $T$ is normal.

The question arises whether the theorem remains valid with normal replaced by subnormal (in both hypothesis and conclusion). The answer is no. Define $T \varphi_{1}=(1 / 4) \varphi_{2}, T \varphi_{2}=(1 / 2) \varphi_{3}, T \varphi_{i}=\varphi_{i+1}$ for $i \geqq 3$. Clearly $T$ is hyponormal, but not subnormal by the previous theorem, We claim $T^{n}$ is subnormal for $n \geqq 2$. For $T^{2} \varphi_{1}=(1 / 8) \varphi_{3}, T^{2} \varphi_{2}=(1 / 2) \varphi_{4}$ 
and $T^{2} \varphi_{i}=\varphi_{i+2}$ for $i \geqq 3$. Thus if we let $g_{j}=\varphi_{2 j-1}$ and $h_{j}=\varphi_{2 j}$ for $j=1,2, \cdots$, then we may write $T^{2}$ as the direct sum of operators acting on $H_{1}=$ span of $\left.g_{j}\right\}$ and $H_{2}=$ span of $\left.h_{j}\right\}$. Now if $F g_{1}=(1 / 8) g_{2}$ and $F_{1} g_{j}=g_{j+1}$ for $j \geqq 2$, then $F_{1}$ is subnormal on $H_{1}$. Similarly, if $F_{2} h_{1}=(1 / 2) h_{2}$ and $F_{2} h_{j}=h_{j+1}$ for $j=2$, then $F_{2}$ is subnormal on $H_{2}$. But $T^{2}$ on $H$ equals $F_{1} \oplus F_{2}$ on $H_{1} \oplus H_{2}$ and hence $T^{2}$ is subnormal. For $n \geqq 3, T^{n}$ can be shown to be subnormal by a similar argument.

We will now exhibit two subnormal operators which are similar but whose (minimal) normal extensions are not. Let $\left\{\varphi_{j}\right\}_{j=1}^{\infty}$ be an orthonormal basis for $H$. Define $T \varphi_{j}=\phi_{j+1}$ for $j=1,2,3, \cdots$, and $S \varphi_{1}=(1 / 2) \varphi_{2}, S \varphi_{j}=\varphi_{j+1}$ for $j=2,3, \cdots$. Then $S$ and $T$ are subnormal by Theorem 6 , they are similar and, in fact, the operator $Q$ given by $Q \varphi_{1}=(1 / 2) \varphi_{1} Q \varphi_{j}=\varphi_{j}$ for $j \geqq 2$, effects the similarity. We claim that the minimal normal extensions are not similar. If they were, they would have to be unitarily equivalent since they are normal. The normal extension of $T$ is unitary while the normal extension of $S$ clearly is not which completes the argument.

\section{BIBLIOGRAPHY}

1. J. Bram, Subnormal operators, Duke Math. J. 22 (1955), 75-94.

2. S. R. Foguel, The relation between a spectral operator and its scalar part, Pacific, J. Math. 8 (1958), 51-65.

3. P. R. Halmos, Normal dilations and extensions of operators, Summa Bras. Math. 2 (1950), 124-134.

4. J. G. Stampfli, Hyponormal operators, Pacific J. Math. 12 (1962), 1453-1458.

5. - Hyponormal operators and spectral density, Trans. Amer. Math. Soc., (to appear).

UNIVERSITY OF MICHIGAN 



\section{PACIFIC JOURNAL OF MATHEMATICS}

\section{EDITORS}

\author{
H. SAMELSON \\ Stanford University \\ Stanford, California \\ R. M. BLUMENTHAL \\ University of Washington \\ Seattle, Washington 98105
}

\author{
*J. DUGUNDJI \\ University of Southern California \\ Los Angeles, California 90007
}

RICHARD ARENS

University of California Los Angeles, California 90024

\section{ASSOCIATE EDITORS}
E. F. BECKENBACH
B. H. NEUMANN
F. WOLF
K. YoSIDA

\section{SUPPORTING INSTITUTIONS}

\author{
UNIVERSITY OF BRITISH COLUMBIA \\ CALIFORNIA INSTITUTE OF TECHNOLOGY \\ UNIVERSITY OF CALIFORNIA \\ MONTANA STATE UNIVERSITY \\ UNIVERSITY OF NEVADA \\ NEW MEXICO STATE UNIVERSITY \\ OREGON STATE UNIVERSITY \\ UNIVERSITY OF OREGON \\ OSAKA UNIVERSITY \\ UNIVERSITY OF SOUTHERN CALIFORNIA
}

\author{
STANFORD UNIVERSITY \\ UNIVERSITY OF TOKYO \\ UNIVERSITY OF UTAH \\ WASHINGTON STATE UNIVERSITY \\ UNIVERSITY OF WASHINGTON \\ * * * * \\ AMERICAN MATHEMATICAL SOCIETY \\ CHEVRON RESEARCH CORPORATION \\ TRW SYSTEMS \\ NAVAL ORDNANCE TEST STATION
}

Mathematical papers intended for publication in the Pacific Journal of Mathematics should be typewritten (double spaced). The first paragraph or two must be capable of being used separately as a synopsis of the entire paper. It should not contain references to the bibliography. Manuscripts may be sent to any one of the four editors. All other communications to the editors should be addressed to the managing editor, Richard Arens at the University of California, Los Angeles, California 90024.

50 reprints per author of each article are furnished free of charge; additional copies may be obtained at cost in multiples of 50 .

The Pacific Journal of Mathematics is published monthly. Effective with Volume 16 the price per volume (3 numbers) is $\$ 8.00$; single issues, $\$ 3.00$. Special price for current issues to individual faculty members of supporting institutions and to individual members of the American Mathematical Society: $\$ 4.00$ per volume; single issues $\$ 1.50$. Back numbers are available.

Subscriptions, orders for back numbers, and changes of address should be sent to Pacific Journal of Mathematics, 103 Highland Boulevard, Berkeley 8, California.

Printed at Kokusai Bunken Insatsusha (International Academic Printing Co., Ltd.), No. 6, 2-chome, Fujimi-cho, Chiyoda-ku, Tokyo, Japan.

\section{PUBLISHED BY PACIFIC JOURNAL OF MATHEMATICS, A NON-PROFIT CORPORATION}

The Supporting Institutions listed above contribute to the cost of publication of this Journal, but they are not owners or publishers and have no responsibility for its content or policies.

* Paul A. White, Acting Editor until J. Dugundji returns. 


\section{Pacific Journal of Mathematics}

\section{Vol. 17, No. 2 \\ February, 1966}

Henry A. Antosiewicz, Boundary value problems for nonlinear ordinary

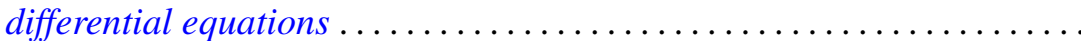

Bernard Werner Levinger and Richard Steven Varga, Minimal Gerschgorin sets. II ........................................

Paul Camion and Alan Jerome Hoffman, On the nonsingularity of complex matrices........................................ 211

J. Chidambaraswamy, Divisibility properties of certain factorials ........ 215

J. Chidambaraswamy, A problem complementary to a problem of Erdös .... 227

John Dauns, Chains of modules with completely reducible quotients ...... 235

Wallace E. Johnson, Existence of half-trajectories in prescribed regions and asymptotic orbital stability ............................ 243

Victor Klee, Paths on polyhedra. II . ........................ 249

Edwin Haena Mookini, Sufficient conditions for an optimal control problem in the calculus of variations ............................ 263

Zane Clinton Motteler, Existence theorems for certain quasi-linear elliptic equations........................................... 279

David Lewis Outcalt, Simple n-associative rings ............... 301

David Joseph Rodabaugh, Some new results on simple algebras ......... 311

Oscar S. Rothaus, Asymptotic properties of groups generation ........... 319

Ernest Edward Shult, Nilpotence of the commutator subgroup in groups admitting fixed point free operator groups .............

William Hall Sills, On absolutely continuous functions and the

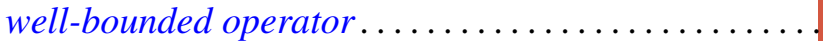

Joseph Gail Stampfli, Which weighted shifts are subnormal ..

Donald Reginald Traylor, Metrizability and completeness in normal Moore spaces 